\title{
Notations and Symbols
}

\section{Object-Orientation}

$\mathcal{L}$

$\mathcal{O}$

$o_{i}$

I

$\mathcal{H}$

$\mathcal{H}_{\mathcal{F}}$

$h_{\mathcal{F}, i}$

$\mathcal{H}_{\mathcal{S}}$

$\mathcal{V}$

$v_{i}$

$\mathcal{C}$

$c_{i}$

$\mathcal{M}$

$m_{i}$

$\mathcal{M}_{n_{i}}$

$\mathcal{O}^{N}$

$\mathcal{O}^{\mathcal{A}}$

$\mathcal{O}^{\mathcal{V}}$

$\tilde{o}_{i}$ object-oriented language based on logic programming

object representation of $\mathcal{L}$

an element (object) of $\mathcal{O}$

set of robot instructions

set of all classes in a class hierarchy

set of fatherclasses

a fatherclass of $\mathcal{H}_{\mathcal{F}}$

set of subclasses

set of instance variables

an element of the set $\mathcal{V}$

set of classes

element of $\mathcal{C}$

set of methods

a method of the set $\mathcal{M}$

the set of methods of the message $n_{i}$

set of object names

set of object names realized as a Prolog atom

set of object names realized as a Prolog variable

element of $\mathcal{O}^{\mathcal{N}}$ 


\section{Concurrency}

$\mathcal{N}$

$n_{i}$

$\mathcal{K}$

$k_{i}$

$\Theta$

$:<>$ :

$:<>$

$:<<:$

$:<<$

$\mathcal{R}$

$r_{i}$

$\Pi$

$\pi$

$A_{\psi}(\ldots)$

$D_{\sigma}(\ldots)$

os

$o_{e}$

$O$ s

$O_{e}$

ᄃ

$a \not \subset b$

$a \rightarrow P$

$(a \rightarrow P \mid b \rightarrow Q)$ set of messages

a message of $\mathcal{N}$

set of channels

a channel of $\mathcal{K}$

set of message passing operators

$S E Q$-operator

$P A R_{I}$-operator

$P A R_{I I}$-operator

$P A R_{I I}$-operator

set of results

a result of $\mathcal{R}$

set of ports

an element (port) of $\Pi$

receiver determination function

distribution process

sender object

receiver object

sender object presented as process

receiver object presented as process

instantiation symbol (is an instance of)

$a$ is not an instance of $b$

$a$ then $p$

$a$ then $P$ choice $b$ then $Q$ (provided $a \neq b$ ) 
$P \| Q \quad \mathrm{P}$ in parallel with $\mathrm{Q}$

$k . v$

communication of value $v$ on channel $k$

\section{Set Theory}

[]

$\phi$

$A \cap B$

$A \cup B$

$A \subseteq B$

$|\mathcal{M}|$

$\bigcap_{n \geq 0} A_{n}$

$\bigcup_{n \geq 0} A_{n}$

$a \backslash b$

$\forall a \in A$

$\exists a \in A$ the empty list

the empty set

$A$ intersect $B$

$A$ union $B$

$A$ is contained in $B$

cardinality of the set $\mathcal{M}$

intersection of family of sets

union of family of sets

$a$ without $b$

for all $a$ in set $A$

there exists an $a$ in set $A$

\section{Sensing}

$\mathcal{B}$

$b_{i}$

$\mathcal{F}$

$\mathcal{F}_{\mathcal{S}}$

$f_{\mathcal{S i}}$

$\mathcal{F}_{\mathcal{R}}$

$f_{\mathcal{R} i}$

$\mathcal{E}_{\mathcal{S}}$ the set of barcodes

a barcode of $\mathcal{B}$

union set of all error codes

the set of system error codes

a system error code of $\mathcal{F}_{\mathcal{S}}$

the set of read error codes

a read error code of $\mathcal{F}_{\mathcal{R}}$

the set of system error messages 
$e_{S i}$

$\mathcal{E}_{\mathcal{R}}$

$e_{\mathcal{R} i}$

\section{Robot}

W

$\Omega^{W}$

$\Omega_{\rho_{i}}$

$\omega_{\rho_{i}, j}$

$\Omega^{\kappa}$

$\iota_{\Omega \times}$

$\Phi_{w}$

$\Phi$ s

$\Phi e$

$\Phi_{p}$

$\left(r_{e}, z_{e}\right)$

O

$O_{n}$

\section{Scheduling}

$\mathcal{A}$

$\mathcal{P}$

$p_{i}$

$\langle\alpha|\beta| \gamma\rangle$

$\alpha$ a system error message of $\mathcal{E}_{\mathcal{S}}$

the set of read error messages

a read error message of $\mathcal{E}_{\mathcal{R}}$

working cell of a robot

overall robot operational space of a working cell $W$

local operational space of robot $\rho_{i}$

position $j$ within the local operational space $\Omega_{\rho_{i}}$

a critical section

list used to manage the queries of a critical section

rotation angle about the $z$-axis of a robot

shoulder rotation angle of a robot

elbow rotation angle of a robot

rotation angle of the wrist pitch of a robot

tool center point $T C P$

coordinate basic origin

transformed coordinate basic origin set of scheduling constraints

set of processors (transputers)

a processor (transputer) of $\mathcal{P}$

specification of a scheduling problem

processor (transputer) characteristics 


\begin{tabular}{|c|c|}
\hline$\beta$ & robot task characteristics \\
\hline$\gamma$ & $\begin{array}{l}\text { optimality criterion (e.g. schedule length minimiza- } \\
\text { tion) }\end{array}$ \\
\hline $\mathcal{T}$ & set of robot tasks \\
\hline$t_{i}$ & a task $t_{i}$ of $\mathcal{T}$ \\
\hline $\mathcal{T}_{\mathcal{A}}$ & the task-dependent subset of $\mathcal{T}$ \\
\hline$t_{a} \prec_{\mathcal{A}} t_{b}$ & task $t_{b}$ is task-dependent from task $t_{a}$ \\
\hline $\mathcal{T}_{\mathcal{D}}$ & the data-dependent subset of $\mathcal{T}$ \\
\hline$t_{a} \prec_{\mathcal{D}} t_{b}$ & task $t_{b}$ is data-dependent from task $t_{a}$ \\
\hline$\tilde{\mathcal{T}}$ & the independent subset of $\mathcal{T}$ \\
\hline$t_{a} \nprec_{A, D} t_{b}$ & task $t_{b}$ is independent from task $t_{a}$ \\
\hline$t^{\prime} \prec t^{\prime \prime}$ & $t^{\prime}$ is the predecessor of $t^{\prime \prime}$ and $t^{\prime \prime}$ is the successor of $t^{\prime}$ \\
\hline$\llbracket p_{i} \rrbracket$ & load of processor $p_{i}$ \\
\hline$t_{k} \leadsto p_{j}$ & task $t_{k}$ is assigned to transputer $p_{j}$ \\
\hline $\mathrm{L}$ & list symbol \\
\hline $\mathrm{E}_{n_{i}}$ & message $n_{i}$ in terms of a list \\
\hline $\mathrm{L}_{\mathcal{D}}$ & a list of data-dependent tasks \\
\hline $\mathrm{E}_{\sigma}$ & list of robot tasks to be scheduled \\
\hline $\mathrm{L}_{S i}$ & a sublist of $\mathrm{L}_{\mathcal{D}}$ \\
\hline$\tau_{i}$ & processing time of task $t_{i}$ \\
\hline$\tau_{\text {idle }}$ & idle time of a processor \\
\hline$\tau_{\sigma}$ & schedule length \\
\hline$\tau_{\sigma, \min }$ & minimized schedule length \\
\hline$\tau_{\sigma_{\text {det }}}$ & determination time of a schedule \\
\hline$t_{i} / n$ & robot tasks $t_{i}$ using $n$ time units to be processed \\
\hline$A\left(P_{S}\right)$ & accuracy of the heuristic scheduling algorithm \\
\hline
\end{tabular}


$P_{S} \quad$ scheduling problem

$S\left(P_{S}\right) \quad$ scheduling result

$O_{P T}\left(P_{S}\right) \quad$ optimal value of the scheduling problem $P_{S}$

\section{Performance}

$S_{p}$

$\tau_{1}$

$\tau_{n}$

E

$T_{\text {post }}$

$T_{\text {mech }}$

$T_{\text {robot }}$

$T_{C S-P r o l o g}$

TPROLoop

$T_{\text {trans }}$

$T_{\text {com }}$

$T_{e x}$

$E_{f}$

$T_{l}$

$T_{c}$

$A P F$

$C\left(P_{S}\right)$

V

$\underline{A}$ speedup

execution time of robot tasks using a single transputer execution time of robot tasks using $n$ transputer

efficiency

translation time used by the postprocessor

time needed by the robot mechanics to perform the tasks

execution time used by a robot system to perform a task

robot task translation time of the CS-Prolog system translation time of the PROLoop of a robot task total translation time of object-oriented robot tasks communication time

total execution time of an object-oriented concurrent robot task

execution factor

data transfer startup time

average transfer time

average parallelism factor

communication cost of a schedule $P_{S}$

volume matrix

assignment matrix 
$\underline{D}$

$v_{i, k}$

$d_{i, j}$

$a_{l, k}$

\section{Miscellaneous}

$\mathcal{G}$

$E_{\mathcal{G}}$

$Z_{\mathcal{g}}$

$a \wedge b$

$a \vee b$

$\mathbb{N}$

$\mathbb{Z}_{0}^{+}$

$F_{\mathcal{G}}$

$O(f(n))$

$\min _{k \in N}\left\{a_{1}, a_{2}, \ldots, a_{k}\right\}$

\# distance matrix

volume of data sent from object $o_{i}$ to object $o_{k}$

communication cost between processor $p_{i}$ and processor $p_{j}$

binary assignment values a directed graph

the set of edges of a graph

the set of vertices of a graph

$a$ and $b$ (logical AND)

$a$ or $b$ (logical OR)

the set of all positive integers

the set of all non-negative integers

a partial order over the set $Z_{\mathscr{G}}$

order of $f(n)$

minimum of the set $\left\{a_{1}, a_{2}, \ldots, a_{k}\right\}$

number of elements 
